\title{
La transición de los estudios de máster: motivaciones y expectativas de estudiantes de dos universidades brasileñas
}

\author{
Tânia Regina Raitz \\ Universidade do Vale do Itajaí. Brasil. \\ raitztania@gmail.com \\ Franciele Corti \\ Universitat de Barcelona. España. \\ fcorti@ub.edu \\ Danilo José Ferreira \\ Universidade do Vale do Itajaí. Brasil. \\ danjosferreira@hotmail.com
}

Alexandre Vanzuita

Instituto Federal de Educação, Ciência e Tecnologia Catarinense. Brasil. alexandre@ifc-camboriu.edu.br

Melissa Zeni

Universidade do Vale do Itajaí. Brasil.

melissa.zeni1@gmail.com

Recibido: 27/10/2017

Aceptado: 4/2/2019

Publicado: 15/7/2019

\section{Resumen}

Este artículo tiene como objetivo estudiar el proceso de transición académica y laboral de los estudiantes del máster en Educación de dos universidades brasileñas - la Universidad de Desarrollo de Santa Catarina y la Universidad del Valle de Itajaí- en cuanto al perfil del alumnado, los factores motivadores para la elección del máster y sus expectativas de formación y de oportunidades laborales. La recogida de datos se realizó mediante la aplicación de un cuestionario. Para la elección de los estudios se identificaron como factores de motivación los requisitos del mercado laboral, la búsqueda de ingreso a la carrera académica y la mejora y la redirección de la trayectoria profesional. Se concluye que el principal beneficio de la realización del máster guarda relación con los compañeros y el ascenso profesional logrado por la obtención del título del máster. Sin embargo, la mayoría de estos alumnos ha pensado abandonar los estudios alguna vez, a causa de la demanda y la presión por la producción académica. Aun así, los encuestados señalan que el máster es una etapa fundamental para su formación y resulta un medio para realizarse profesionalmente.

Palabras clave: transición académica y laboral; máster en Educación; motivación; expectativas 
Resum. La transició dels estudis de màster: motivacions i expectatives d'estudiants de dues universitats brasileres

Aquest article té com a objectiu investigar el procés de transició acadèmica i laboral dels estudiants del màster en Educació de dues universitats brasileres - la Universitat de Desenvolupament de Santa Catarina i la Universitat de la Vall d'Itajaí- quant al perfil de l'alumnat, els factors motivadors per a l'elecció del màster i les expectatives dels estudiants quant a la formació i les oportunitats laborals. La recollida de dades es va realitzar mitjançant l'aplicació d'un qüestionari. Per a l'elecció dels estudis de màster es van identificar com a factors de motivació els requisits del mercat laboral i la persecució d'una carrera acadèmica, així com la millora i la redirecció de la trajectòria professional. El text conclou dient que el principal benefici de la realització del màster per als estudiants és la relació establerta amb els companys i l'ascens professional aconseguit per obtenir el títol corresponent. No obstant això, la majoria d'aquests alumnes ha pensat abandonar els estudis alguna vegada, a causa de la demanda i la pressió per producció acadèmica. Tot i així, els enquestats assenyalen que el màster és una etapa fonamental per a la seva formació i també un mitjà per realitzar-se professionalment.

Paraules clau: transició acadèmica i laboral; màster en Educació; motivació; expectatives

\section{Abstract. Transition from master's degree programs: Motivation and expectations of students at two Brazilian universities}

The aim of this article is to investigate the academic and labor transition process of students in master's degree programs in education at two Brazilian universities: Universidade do Desenvolvimento de Santa Catarina and Universidade do Vale do Itajaí. To this end, student profiles, the factors that motivate them to choose the program, and their expectations regarding training and labor opportunities are explored. Data were collected by means of a questionnaire with open and closed questions. In both groups, labor market requirements, pursuing an academic career, and improving and reorienting career paths were identified as motivating factors for entering the master's programs. Based on the survey data, it is concluded that relationships with colleagues and professional growth were the main benefits of completing the degree. However, the majority of students considered dropping out at some point due to the demanding requirements of the courses. Even so, the respondents indicated that the master's degree is an essential stage of their training and a means to achieving professional fulfillment.

Keywords: academic and employment transition; master's programs in education; motivation; expectations

\section{Sumario}

\section{Introducción}

2. Estudios sobre las transiciones académicas y laborales en la formación de máster

3. Metodología: objetivos, sujetos, instrumento de recogida de datos y análisis
4. Resultados del estudio: perfil, motivaciones y expectativas de los estudiantes

\section{Conclusiones}

Referencias bibliográficas 


\section{Introducción}

La situación brasileña actual, tanto por lo que se refiere a los aspectos económico y productivo como a las vertientes política, social y cultural, se refleja en el ámbito educativo, que no escapa a las contingencias del momento complejo que vivimos. Aun así, de acuerdo con el Plan Nacional de PostGrado (PNPG) (CAPES, 2010), el país entró en el siglo XXI como una nueva potencia emergente, con la perspectiva de llegar a ser la quinta economía del mundo en la década de 2011-2020.

En los últimos años, las políticas sectoriales en Brasil han llevado a una fuerte expansión de los programas de postgrado. Se han creado nuevas universidades federales — públicas y gratuitas - y estudios de máster en instituciones educativas privadas (Morosini, 2009). En consonancia, ha crecido significativamente el volumen de estudiantes de posgrado en el país. Sin embargo, el número de titulados en máster es aún insuficiente si se compara con otras potencias mundiales.

Así, el país viene desarrollando una política orientada al acceso a los estudios de máster impulsada por los planes nacionales de estudios de postgrado (PNPG). Está en marcha el VI PNPG (2011-2020), que tiene como objetivo prioritario el crecimiento continuo de la calidad del Sistema Nacional de Postgrado. El impacto es tan importante que incluso desde los sectores privados del mercado laboral se potencia la formación y la obtención del título de máster dentro de su plantilla de trabajadores.

Según la CAPES (Coordinación de Perfeccionamiento del Personal Universitario), en el transcurso de 2004 y 2005 había 72.000 alumnos matriculados en la formación de segundo ciclo (CAPES 2010). Velloso (2014) añade que en 25 años — desde 1987 hasta 2012 - el número de estudiantes titulados en máster aumentó de 861 a 42.878, lo que representa un incremento considerable. En los últimos 10 años se ha aumentado la oferta de programas de postgrado stricto sensu (másteres universitarios y doctorados) en el ámbito de la educación en Brasil: de 36 a 86 programas (un incremento de más del 138\% en tan solo una década), lo que, según Velloso (2014: 12), «es una clara indicación del desarrollo del sector, que ha ido cambiando y, con eso, atrayendo a profesionales no solo del ámbito educativo, sino también de otras áreas del conocimiento".

Avanzando en la delimitación de nuestro tema de estudio, a través de un convenio entre Brasil y España, en concreto entre la Universidad de Barcelona (UB) y la Universidad del Valle de Itajaí (UNIVALI), se ha desarrollado un proyecto DGU/CAPES que ha permitido reflexionar sobre los procesos de transición de los estudios de máster en el ámbito de la educación entre los dos países. Sin embargo, en el presente artículo presentaremos solamente los resultados de la investigación en dos universidades brasileñas: la Universidad de Desarrollo de Santa Catarina (UDESC) y la Universidad del Valle de Itajaí (UNIVALI). Más allá de la expansión cuantitativa de programas de postgrado verificada en el país, los resultados del presente análisis confirman la existencia 
de algunas diferencias en las tipologías de estudiantes que acceden a la formación de máster en Educación, si lo comparamos con el contexto español. En Brasil, el perfil mayoritario de estos estudiantes es el de profesionales de más de 30 años que cuentan con una experiencia laboral reconocida, que buscan una promoción en su puesto laboral y una sólida formación teórica a través de la obtención del título de máster.

De este perfil se desprende que se está potenciando la formación de máster de carácter eminentemente profesional. Así, las personas que se matriculan en dichos estudios lo hacen, mayoritariamente, con la finalidad de acceder a una carrera académica y ampliar sus conocimientos, así como conseguir una progresión laboral. El análisis cubre una brecha en la investigación sobre los factores de motivación y las expectativas de los alumnos matriculados en másteres en el área de la educación. De ahí su importancia, puesto que tiene en cuenta la tipología, las motivaciones y las expectativas de los estudiantes de máster en Brasil, fundamentada en el proceso de transición que viven ellos.

La evolución de las trayectorias lleva a reflexionar sobre la formación académica del alumnado de máster como una nueva experiencia de aprendizaje que guía su conducta futura. Investigar los factores de motivación y las expectativas que conducen a los estudiantes de primer año a elegir estos estudios es esencial para que las instituciones educativas puedan satisfacer sus expectativas y evitar la frustración y la desilusión por parte de aquellos.

\section{Estudios sobre las transiciones académicas y laborales en la formación de máster}

La búsqueda de mejora o la continuación de sus estudios, ya sea a través de una especialización profesional o de estudios de máster académico, es una etapa que se convierte en un momento crítico, de toma de decisión. El proceso de elección de un estudio de máster conducirá inevitablemente a nuevas relaciones, a la profesionalización como investigador novel, a la construcción de una nueva identidad profesional y a la puesta en práctica de nuevos conocimientos en su área de especialización.

Benites y Neto (2011) consideran que los factores intervinientes en la elección de un máster son la noción de continuidad de la formación inicial, las necesidades de la profesión en el mercado de trabajo, el estatus social de la profesión y la familia. También aparecen en la bibliografía especializada otros dos factores: el entorno social y el momento histórico.

El momento de la elección de carrera es abordado por Valério y Souza (2012), quienes reafirman los elementos fundamentales en esa toma de decisión: características personales, habilidades específicas en un área en particular, contexto histórico y entorno sociocultural del sujeto. La elección de los estudios también está íntimamente relacionada con lo que estos pueden aportar para el desarrollo de habilidades y competencias que guían las motivaciones de los estudiantes. La opción por un estudio de máster está influenciada por una serie de factores relacionados con la personalidad; la familia; los miedos; 
el entorno social, político y cultural; los medios de comunicación, y el proceso educativo.

Para verificar si los estudios de máster contribuyen a seguir una trayectoria de éxito y coherente con su desempeño en el mercado laboral, es fundamental, por lo tanto, conocer la experiencia formativa de estos alumnos. Los estudios de los procesos de transición han recibido un impulso en las últimas décadas, lo que favorece la aparición de un corpus teórico en torno a este constructo. En consonancia con la incertidumbre de los tiempos modernos, los estudios sobre la formación recibida, el trabajo y los proyectos futuros de los titulados en másteres están adquiriendo una gran importancia actualmente. La temática preocupa a los académicos y a los profesionales de la inserción sociolaboral, el proyecto profesional de carrera y la orientación profesional.

A partir de las transformaciones laborales que tuvieron lugar en la década de 1990, emergió un nuevo paradigma del trabajo. Más allá de la perspectiva de formalidad laboral, que se expresaba por la pérdida de sentido y la devaluación de la tarea profesional, esta nueva perspectiva causó decepción en los sujetos de la sociedad contemporánea, pues ya no cumplía con sus expectativas. En este sentido, hubo un cambio en las concepciones de sujeto e identidad laboral (Bendassolli, 2007).

Es esencial tener en cuenta la situación actual de las estructuras de trabajo, que están sujetas a rápidos cambios, en la forma como los jóvenes experimentan esta transición entre la universidad y el mercado laboral. En períodos de crisis drásticas, el proceso de transición está influido no solamente por los cambios económicos, sino también por el desarrollo tecnológico, en combinación con los procesos de globalización de la economía. Todo ello ejerce un impacto significativo en la lógica de la producción y la organización del trabajo; además de la flexibilidad del mercado laboral, que afecta a las formas de contratación. Rodríguez-Moreno, Álvarez, Figuera Gazo y Rodríguez-Espinar (2008) sugieren que las tendencias globales presentan un mercado de trabajo que no es uniforme, por lo que las variaciones (en cuanto a los ciclos económicos y a la lógica de producción y organización del trabajo) no afectan de igual manera a todos los niveles de estratos.

Figuera (1996) analiza la inserción profesional desde una perspectiva psicosocial, a partir de un estudio con graduados de la Universidad de Barcelona. La calidad de la inserción de los titulados universitarios establece un indicador para evaluar en qué medida los estudios superiores cumplen su emergente función profesionalizadora. Los datos indican que el proceso de inserción tiene sus raíces en la naturaleza interactiva de las características personales y contextuales que le confieren su carácter de fenómeno psicosocial y representa la síntesis de la proyección humana: individual y social.

En este sentido, en Brasil existe una clara reducción en la oferta de puestos de trabajo formales, especialmente para los jóvenes. Se trata de un mercado muy exigente con las habilidades y las competencias de las personas, que terminan por culpabilizar esta exigencia por el éxito o el fracaso de su experiencia de transición. Sin lugar a dudas, esta situación está estrechamente relacionada 
con otro fenómeno que se ha convertido en un tema recurrente de los centros de formación: la deserción universitaria.

Focalizando la mirada en los estudios de postgrado, la investigación de Figuera, Buxarrais, Llanes y Venceslao (2018) analiza el perfil, la motivación y la satisfacción académica en los estudios de máster de Ciencias Sociales y Jurídicas de la Universidad de Barcelona, con la participación de 453 estudiantes provenientes de 8 másteres de la Universidad de Barcelona y de la Universidad Autónoma de Barcelona. Los resultados muestran diferencias entre la tipología de máster y los motivos de elección de la formación, así como en la satisfacción con los estudios. Se confirma la especificidad de los procesos de transición a estos estudios y la importancia de establecer acciones orientadoras.

En el contexto brasileño, Silva y Bardagi (2015) señalan que, debido al creciente número de estudiantes matriculados en postgrados, ha aumentado en los últimos años la voluntad de entender las elecciones, las expectativas y las dificultades de esos estudiantes. Una de las principales conclusiones del estudio es la necesidad de realizar investigaciones enfocadas al desarrollo de carreras y a la adaptación académica de los postgraduados (Silva y Bardagi, 2015). Además, se recomienda una diversificación en las áreas de estudio, de acuerdo con la especificidad de la formación de cada área.

Además de los estudios de máster, estas mismas autoras también abordan la formación continuada en general (cursos de especialización y másteres propios). Desde el año 2000 se verifica en Brasil un aumento considerable en todas estas modalidades de formación, lo que lleva a considerar la importancia de la transición entre graduación y postgrado. Silva y Bardagi (2015) señalan que esta elección se relaciona, en general, con una búsqueda de cambio en el área de actuación laboral y con un aumento del interés por el desarrollo de la carrera. Finalmente, argumentan que el crecimiento de la población universitaria y de los postgrados configura un nuevo perfil de usuarios para los programas de orientación profesional.

Si nos centramos en el contexto anglosajón, son escasos los análisis que se centran en el estudio de la transición al nivel de postgrado. Hurtado (2018) señala que las principales y más amplias investigaciones realizadas en Estados Unidos acerca de la vida de los estudiantes y las prácticas educativas universitarias, en general, no consideran a los alumnos de postgrado en sus muestras de estudio. En el contexto europeo, una investigación reseñable en el estudio de las transiciones académicas y en la búsqueda de entendimiento de las cuestiones relacionadas al alumnado es el proyecto EUROSTUDENT. Su finalidad es recopilar datos comparables sobre la dimensión social de la educación superior en Europa. Sin embargo, el postgrado tampoco ha sido, hasta la actualidad, un foco de interés (Hauschildt, 2018).

De lo expuesto hasta el momento se desprende la necesidad de realizar investigaciones que tengan como temática el alumnado de máster y como objetivo identificar los factores de motivación para la elección del máster, conocer la percepción del alumnado de los beneficios y de las dificultades 
asociadas a la formación realizada e informarse sobre la expectativa del alumnado respecto a las oportunidades laborales que podrán generarse a raíz de la realización del máster.

\section{Metodología: objetivos, sujetos, instrumento de recogida de datos $\mathrm{y}$ análisis}

El objetivo general de la presente investigación es estudiar el proceso de transición académica y laboral de los estudiantes de máster en Educación de dos universidades brasileñas: la Universidad de Desarrollo de Santa Catarina (UDESC) y la Universidad del Valle de Itajaí (UNIVALI). Los objetivos específicos son los siguientes:

a) Describir el perfil del alumnado del máster en Educación.

b) Identificar los factores motivadores para la elección del máster.

c) Conocer la percepción del alumnado acerca de los beneficios y las dificultades asociados a la formación realizada.

d) Conocer las expectativas del alumnado respecto a las oportunidades laborales que podrán generarse a raíz de la realización del máster.

Se trata de una investigación cuantitativo-descriptiva y exploratoria en la que se utilizó un cuestionario con 25 preguntas abiertas y cerradas, con la finalidad de entender los fenómenos y las tendencias objeto de investigación. El cuestionario fue creado ad hoc a partir de las aportaciones teóricas. Las dimensiones que componen el instrumento son: indicadores sociodemográficos, procedencia académica, trayectoria laboral, proceso de elección del máster, proyecto profesional, satisfacción académica, factores de transición y expectativas de futuro. El instrumento pasó por un proceso de validación a través de una aplicación piloto.

Los sujetos que componen la población del análisis son los alumnos matriculados en los másteres en Educación de las dos universidades brasileñas, lo que nos permite relacionar los factores socioculturales en la elección de los estudios, el acceso, las motivaciones y las expectativas ante el mismo. Todos los matriculados en ambos programas de máster — 89 estudiantes - fueron invitados a participar en la investigación. La muestra resultante está compuesta por los 47 sujetos que se presentaron voluntariamente. El perfil de estos estudiantes se expondrá en el siguiente apartado.

Para el análisis de datos, se realizó un examen estadístico a través del software SPSS. Las preguntas abiertas del cuestionario fueron observadas a partir de la técnica de análisis de contenido basado en Bardin (2010), en el que el análisis interpretativo, las inferencias, las constancias y las recurrencias sirvieron para dar respuesta al objetivo del estudio. En el presente texto nos centraremos sobre todo en los datos cuantitativos, mientras que la información cualitativa servirá para complementar y matizar los datos estadísticos. 


\section{Resultados del estudio: perfil, motivaciones y expectativas de los estudiantes}

A continuación presentamos los resultados de la investigación distribuidos en tres categorías de análisis: $a$ ) perfil de los estudiantes, $b$ ) motivaciones para acceder al máster y $c$ ) expectativas que estos estudiantes tienen en relación con la formación de los estudios de máster en Educación y su vida laboral.

\section{a) Perfil de los estudiantes}

La muestra total es de 47 estudiantes y está compuesta por 17 alumnos del máster en Educación de la UDESC - de una población total de 39 matriculados (un 37,5\%) - y por 30 estudiantes del máster en Educación de la UNIVALI - de una población total de 50 matriculados (un 62,5\%) — (véase la figura 1). Se ha optado por investigar un máster de una universidad pública (UDESC) y uno de una universidad privada (UNIVALI).

Se observa que el $24,4 \%$ de la muestra tuvo que mudarse de ciudad para poder realizar los estudios de máster y que el $75,6 \%$ no tuvo que cambiar de residencia. Por lo general, este factor es importante, porque los desplazamientos de población siempre implican un incremento en su presupuesto. Con respecto al género, el $77,1 \%$ son mujeres y el $22,9 \%$ son hombres. Hay un predominio de mujeres que trabajan en la educación, lo que promueve que esta se asocie más al género femenino. Por supuesto esta imagen está cambiando con el tiempo, pero conceptos como cuidado y maternal aún se relacionan con este ámbito.

Acerca de la distribución etaria, la muestra es dispersa, puesto que abarca desde los 21 hasta los 63 años, con una mayor concentración entre los 32 y los 42 años. El perfil mayoritario de los estudiantes es profesional, con más de

Figura 1. Tipología de centro de realización del máster $(n=47)$

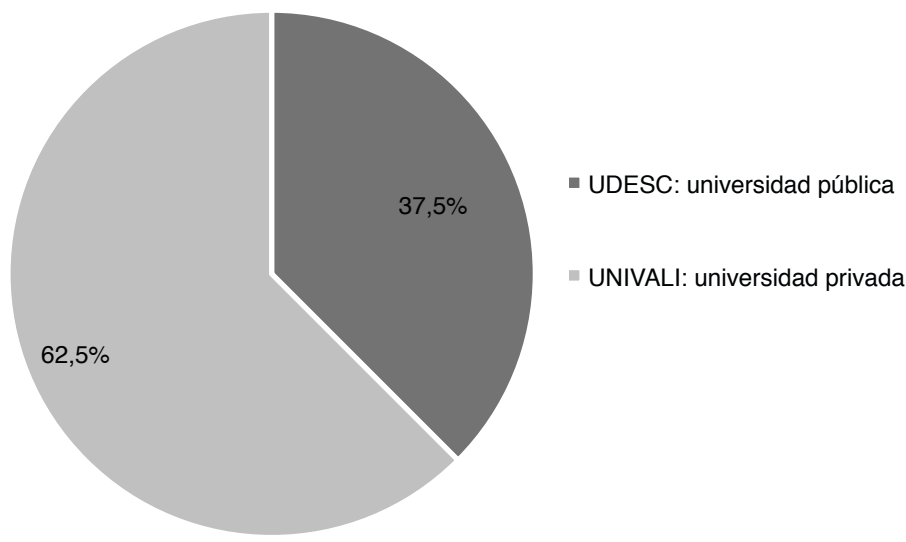

Fuente: elaboración propia. 
32 años de edad y con una experiencia laboral reconocida, que busca con la obtención del título del máster una mejora en su puesto laboral y una sólida formación teórica.

Respecto a la situación familiar, se detecta que el 36,4\% de la muestra vive con los padres, el 29,5\% es independiente con hijos, el 22,7\% es independiente y no tiene hijos y el $11,4 \%$ vive con otros parientes (que no son padres o hijos).

La situación laboral de los estudiantes se presenta en la figura 2, donde se observa que el 52,3\% de la muestra trabaja a jornada completa, lo que probablemente influye en la percepción de los alumnos de que el máster les exige demasiado. La dificultad de compaginar trabajo y formación y el sentimiento de agobio que se genera a partir de esta situación es uno de los motivos para pensar abandonar los estudios. En cambio, el 20,5\% de la muestra está en situación de paro, afectado por la crisis y el contexto de cambio en el mercado de trabajo. Un contexto laboral heterogéneo genera una recesión que impulsa a los estudiantes a incrementar sus credenciales de empleabilidad (Alfaro, 2009; Bendassolli, 2007; Rodríguez-Moreno et al., 2008).

En lo que se refiere a la experiencia de trabajo, se observa que el 56,4\% de los estudiantes son recién titulados, lo que se correlaciona directamente con la disminución de la media de edad general. El 7,7\% de los alumnos tiene menos de 5 años de experiencia laboral (experiencia júnior) y el 35,9\% tiene más de 5 años de experiencia laboral (experiencia sénior). Si tenemos en cuenta la tipología de universidad, se observa que la mayoría de los estudiantes con menos experiencia laboral están matriculados en la universidad privada (un 66,7\%).

Figura 2. Situación laboral de los estudiantes del máster $(n=42)$

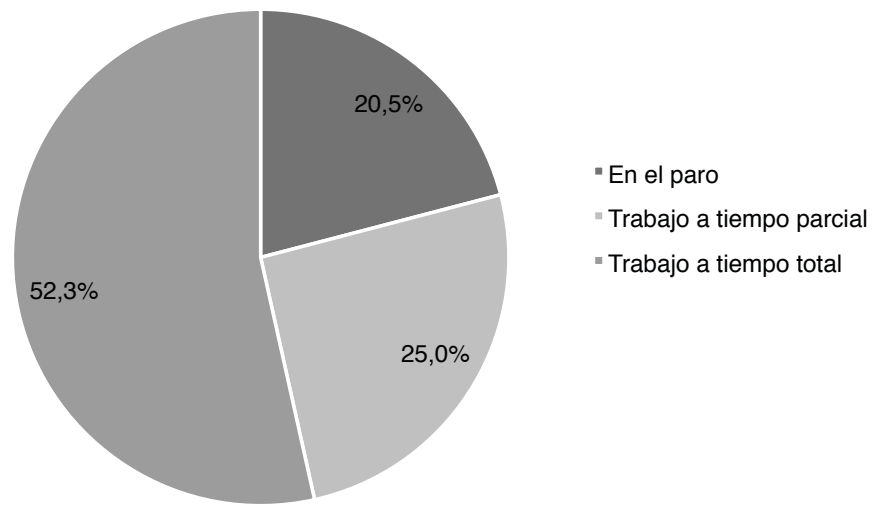

Fuente: elaboración propia ${ }^{1}$.

1. Es importante señalar que la muestra $(n)$ de cada pregunta no siempre coincide con la muestra total del estudio. En el caso de la pregunta presentada en la figura 2 contamos específicamente con 42 respuestas. 


\section{b) Motivaciones para acceder al máster en Educación}

Como se puede observar en la figura 3, la necesidad de obtener una especialización profesional fue el principal motivo para realizar el máster, apuntado por el 50\% de los encuestados; el cambio de área de actuación profesional es el motivo señalado por el $25 \%$ de los sujetos; la promoción o el acenso laboral representa el motivo del 20,5\% de ellos, y tan solo el 4,5\% indica querer redefinir su trayectoria profesional. Se ha observado que los dos grupos de estudiantes - UDESC y UNIVALI- afirman que la formación a nivel de máster fomentará sus oportunidades de trabajo, o bien para mantenerse en su empleo o bien para introducirse en el mercado laboral.

Los sujetos reconocen que la demanda de titulación está influenciada por la importancia que el país atribuye a la educación permanente o continua como garantía de obtener un empleo, en base a la creencia generalizada de que una mayor formación les permitirá — en consonancia con la tesis de la teoría del capital humano (Schultz, 1963) — conseguir mejores oportunidades laborales, como ya hemos establecido en la parte teórica de este estudio. Ellos entienden la lógica de los mercados competitivos y la demanda de formación como garantía de futuro a partir de una perspectiva mercadológica (Pochman, 2006; Kunzer, 2005). Por lo tanto, en este contexto, la educación continua es la forma ideal para permanecer inserto en el mercado laboral.

En la figura 4 se observa que los recién graduados buscan el acceso al máster para especializarse (un 55,6\%) o para cambiar su área de actuación (un $33,3 \%$ ). De ello se desprende que las principales razones de este colectivo para acceder al máster están relacionadas con las intenciones de ampliar la base teórica adquirida en el grado, incrementar sus habilidades y ascender al programa de doctorado. En cambio, para los estudiantes con menos de 5 años de experiencia laboral, los tres principales motivos de acceso al máster son el reajuste del diseño de su trayectoria profesional, el cambio de área de actuación

Figura 3. Principal razón para acceder al máster $(n=44)$

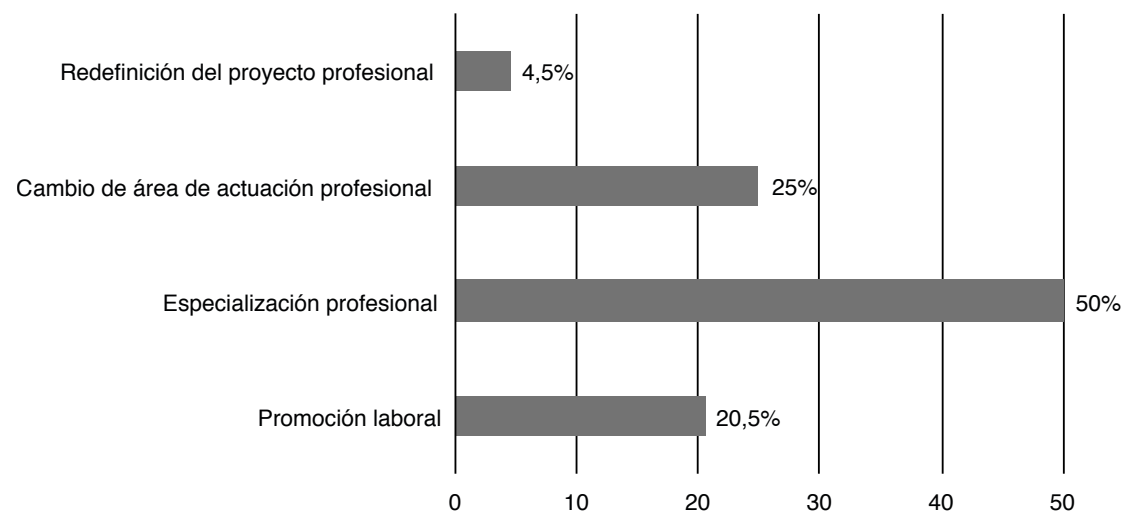

Fuente: elaboración propia. 
y la búsqueda de especialización (todos con un 33,3\%). Los estudiantes que cuentan con más de 5 años de experiencia laboral señalan como principal motivo para acceder a los estudios de máster la búsqueda de especialización (un 50\%) y, en segundo lugar, la promoción en su trabajo y, consecuentemente, un aumento salarial (un 35,7\%). Se puede afirmar que, para las tres tipologías de estudiantes, la búsqueda de especialización es un factor importante para decidirse a realizar los estudios de máster. Estos datos van en consonancia con las aportaciones de Benites, Sarti y Neto (2015), que consideran que la noción de continuidad de la formación inicial, las necesidades de conocer bien la profesión y una mayor cualificación son factores intervinientes en la toma de decisión de realizar un postgrado.

En ese contexto, la formación continua sigue siendo la forma ideal para establecerse y permanecer inserto en el mercado laboral. En Brasil se está impulsando, actualmente, desde las esferas pública y privada del sector laboral, la formación continua de sus trabajadores. Los brasileños conciben la formación como una posibilidad de promocionarse laboralmente y de ascender socialmente. Desde esta perspectiva, se puede visualizar el impacto de las polí-

Figura 4. Principal razón para la realización del máster $(n=44)$

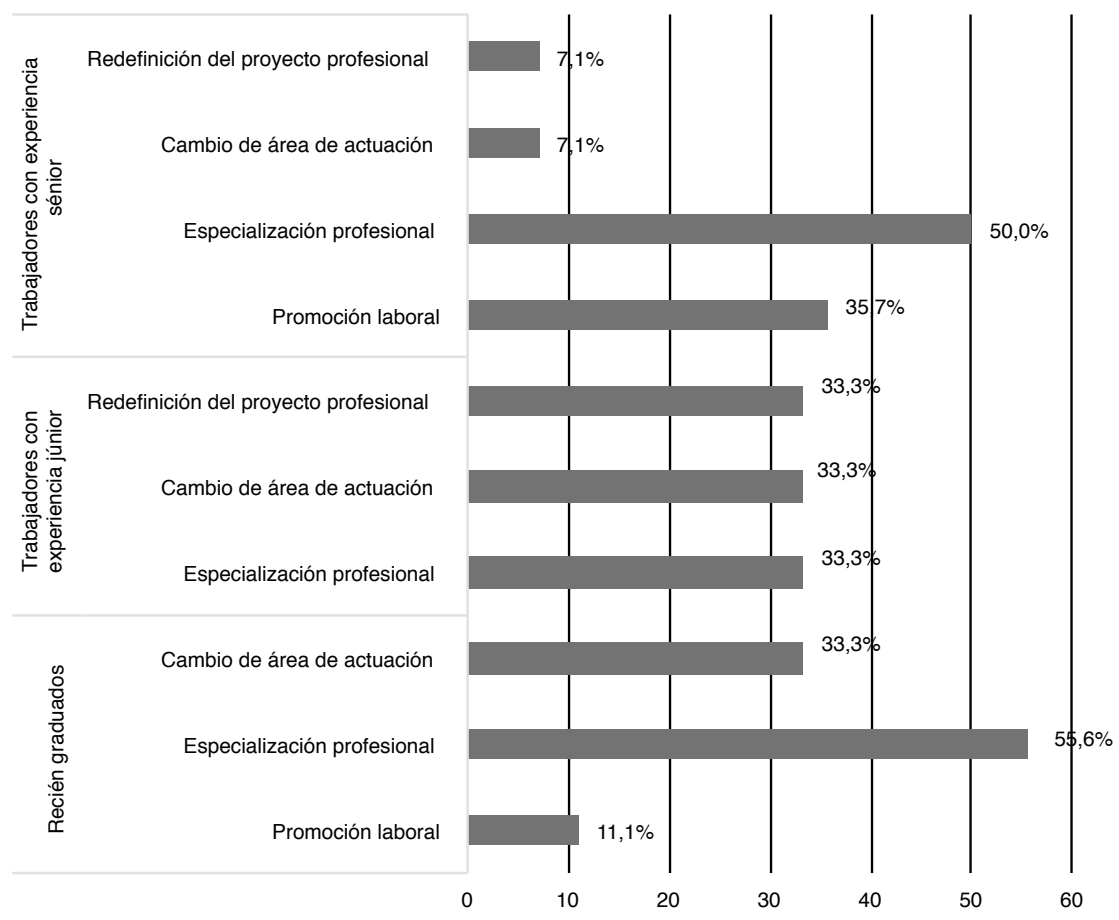

Fuente: elaboración propia. 
ticas públicas en un nivel macro y en la vida personal de los estudiantes de máster (Figuera, 1996).

A partir de los planes actuales que guían las políticas de postgrado en Brasil (CAPES, 2010), se observan mejores expectativas económicas para el país en cuanto a las políticas de formación y se reconoce la formación a todos los niveles, de modo que se otorga un valor añadido a los másteres como potencial diferenciador de ascenso al mercado de trabajo, a la vez que es necesario tener experiencia profesional para ascender laboralmente, ya que las mismas políticas de acceso a los másteres así lo determinan. Por ello se puede afirmar que, en el caso de Brasil, hay una fuerte relación entre la universidad y el mercado de trabajo.

Sin embargo, se ha podido ver que los principales factores de motivación para matricularse en el máster son las demandas del mundo laboral (necesidad de titulación, aumento de empleabilidad y mejores salarios) y la carrera académica, así como la redirección y la mejora profesional (especialización en su área de actuación). Las diferentes perspectivas motivacionales desafían las universidades a reflexionar sobre las condiciones, los procesos y las prácticas que pueden aumentar el éxito de los estudios de máster. Es fundamental conocer la experiencia formativa de estos alumnos, con el fin de verificar si los estudios de máster pueden contribuir a trazar una trayectoria de éxito que sea coherente con su desempeño en el mercado laboral (Silva y Bardagi, 2015).

\section{c) Expectativas respecto al máster en Educación}

Se ha observado que existe un alargamiento de la formación profesional inicial de los alumnos y un aumento en las tasas de regreso a la universidad por parte de profesionales que buscan mejorar sus competencias a través de estudios de máster. Por lo tanto, es importante tener en cuenta la relación que establecen los estudiantes de máster entre la formación recibida y su satisfacción académica. Tal como se puede observar en la figura 5, de una manera general, los encuestados están bastante satisfechos con el máster. En una escala de 1 a 5 puntos califican con un 4,64 su satisfacción con la elección de los estudios, con un 4,57 el estar disfrutando de los estudios, con un 4,51 el sentirse bien con el grupo de compañeros y con un 4,51 el gusto por lo que están aprendiendo.

Sin embargo, verificamos la existencia de una condición paradójica, ya que el $87 \%$ de estos mismos alumnos — que comentan estar satisfechos con los estudios de máster - han pensado o piensan abandonar los estudios. En este sentido, existe una incongruencia entre la satisfacción y las dificultades encontradas y el pensamiento de abandono, lo que también se verifica en el estudio de Figuera et al. (2018).

Un número elevado de encuestados menciona que uno de los aspectos negativos de los estudios es el alto nivel de exigencia del profesorado. En la figura 6 se puede observar que el $100 \%$ de los alumnos con poca experiencia laboral ya ha pensado renunciar a los estudios de máster, así como que el $94,7 \%$ de los graduados recientes y el $69,2 \%$ de los trabajadores con más experiencia han pensado lo mismo. La diferencia entre los índices de los tres 
Figura 5. Satisfacción académica $(n=46)$

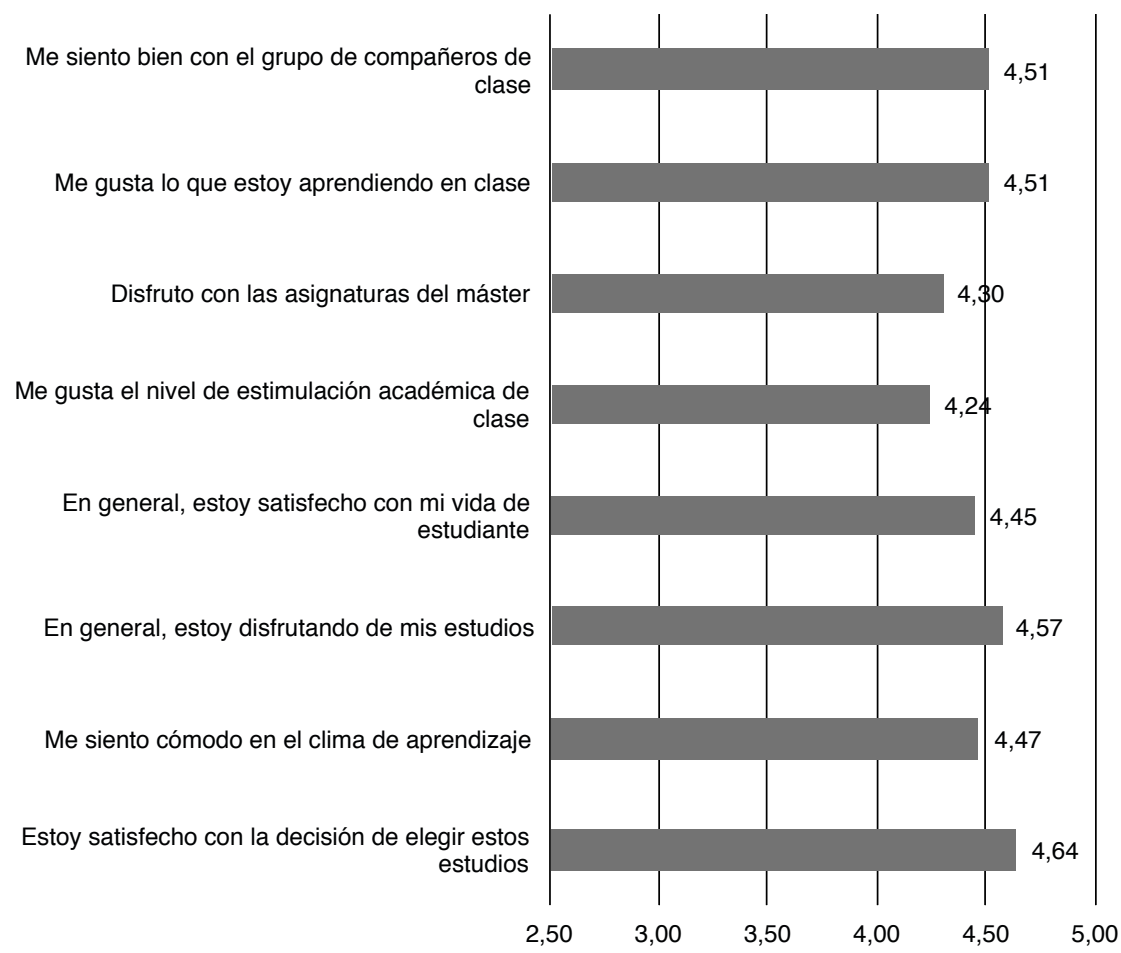

Fuente: elaboración propia.

colectivos puede venir dada por la creencia de una ascensión laboral decurrente en referencia a la adquisición del título de máster por parte de los trabajadores sénior.

Los estudiantes revelan, además, las dificultades a las que se enfrentan durante el período de realización de sus estudios. En una escala del 1 al 7 , siendo 1 el puntaje que indica mayor dificultad y 7 mayor facilidad, los encuestados manifiestan que, a lo largo del máster, han sufrido problemas económicos (4,23 puntos de media) y se ha producido algún evento personal o familiar $(4,34)$ que ha impedido la realización normal del curso. Además, consideran que el desarrollo de la docencia ha influido negativamente en su motivación respecto a los estudios $(4,73)$ y, sobre todo, indican el grado de exigencia de los estudios como principal factor de dificultad en la transición al máster $(3,57)$. Estos factores contribuyen a menudo a hacerles pensar en abandonarlo (véase figura 7).

Los análisis del grupo de investigación sobre transiciones académicas y laborales (TRALS-UB) se destacan como referencia internacional y vienen avanzando en el desarrollo de un modelo de transición que se caracteriza por la integración y la interacción de factores personales e institucionales en la 
Figura 6. Pensamiento de abandono $(n=44)$

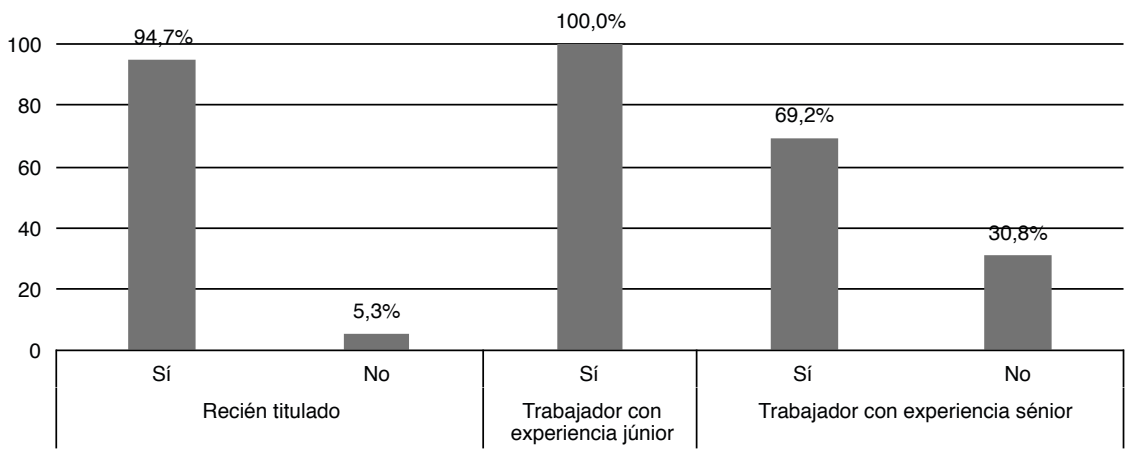

Fuente: elaboración propia.

Figura 7. Factores de transición académica $(n=46)$

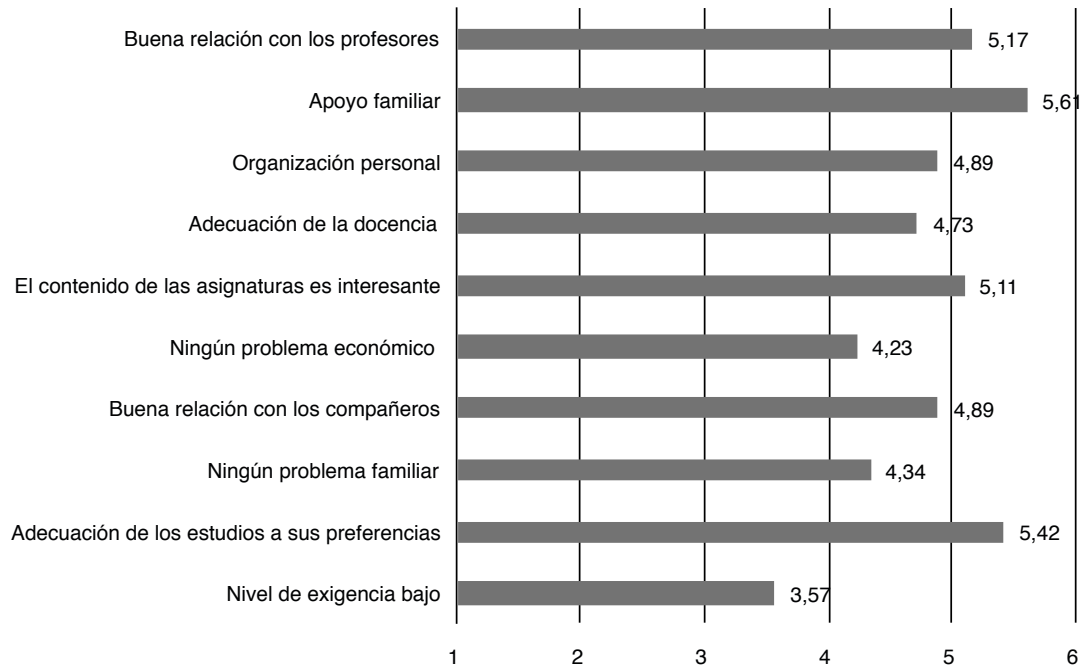

Fuente: elaboración propia.

explicación de los procesos de transición académica y laboral. Las investigaciones del grupo han considerado que la procedencia del estudiante cuando accede a la universidad ejerce influencia en su permanencia en el sistema universitario. De esta manera, consideramos los mismos factores como facilitadores e inhibidores de la permanencia en los estudios de máster: sus antecedentes académicos previos, el entorno familiar y las características personales, el compromiso inicial del alumno en finalizar sus estudios y las interacciones 
positivas con su entorno. Otras investigaciones nacionales e internacionales (como la de Î́niguez, Elboj y Valero, 2016) también han identificado una serie de factores de abandono o persistencia educativa.

\section{Conclusiones}

Una vez presentados los resultados, revisitaremos los objetivos de la investigación para concluir el presente texto. En lo que se refiere a la descripción del perfil del alumnado del máster en Educación, podemos afirmar que nos encontramos con una tipología de estudiantes de máster que, a grandes rasgos, no ha tenido que desplazarse a otra ciudad para realizar sus estudios y que en su mayoría se trata de mujeres con edades entre los 32 y los 42 años.

La mayoría de los estudiantes está trabajando, lo que puede llegar a ser un factor desalentador debido a las altas demandas del máster. Una pequeña parte está experimentando la situación de paro. En ese sentido, el contexto de cambio en el mundo del trabajo actual, el proceso de globalización y la crisis mundial aparecen como impulsores de este proceso.

Acerca del objetivo relacionado con la identificación de los factores motivadores para la elección del máster se encuentran las demandas del mercado de trabajo (Benites y Neto, 2011) (necesidad de titulación, aumento de la empleabilidad y mejores salarios) y el ingreso en la carrera académica, así como una redirección y una mejora de la carrera profesional (especialización en su área de actuación).

En relación con los beneficios proporcionados por la realización del máster, los factores más indicados guardan relación con los compañeros de clase y el ascenso profesional logrado con la obtención del título. Sin embargo, si nos centramos en las dificultades asociadas a la formación realizada, un porcentaje alto de alumnos ya ha pensado o piensa abandonar los estudios, sobre todo por la exigencia de producción y la dificultad para conciliar la formación con el trabajo. En definitiva, el principal hallazgo de este estudio exploratorio es el grado de influencia que las condiciones del mercado laboral ejercen en el perfil, en las aspiraciones, en las expectativas y en el sentido que tiene la formación y el trabajo para los nuevos estudiantes universitarios (Bendassolli, 2007).

Respecto al último objetivo específico, buscamos conocer las expectativas del alumnado respecto a las oportunidades laborales que podrán generarse a raíz de la realización del máster. Se concluye que para estos estudiantes el máster constituye una etapa fundamental para su formación y para el trabajo que quieren desarrollar. Los resultados demuestran que, en la realidad brasileña, el máster es un medio para realizarse profesionalmente (Luna, Santos y Bardagi, 2014). Dicha formación constituye un proceso complejo que involucra no solo a la participación de los estudiantes, sino también de profesores y de la propia institución de enseñanza a través de una adecuada estructura organizativa. Este proceso de transición requiere adaptaciones y ajustes de todos los involucrados. Es en esta compleja realidad socioeducativa que la 
orientación se convierte en un valor añadido de desarrollo integral del alumno y en la propia mejora de la institución educativa (Silva y Bardagi, 2015).

Finalmente, es reseñable la baja frecuencia de análisis centrados en los estudiantes de máster, tanto en el entorno anglosajón como en el español y en el brasileño. De esta manera se sugiere, como línea prospectiva, una profundización desde la óptica de la orientación profesional en las temáticas relacionadas con la elección de los másteres, las motivaciones y las expectativas del alumnado y la cualificación profesional, las oportunidades laborales y las trayectorias de los egresados de los másteres (Méndez Rebolledo, 2018).

\section{Referencias bibliográficas}

Alfaro, I. (2009). Diagnóstico para las transiciones académico-profesionales. En L.M. Sobrado y A. Cortéz (coords.). Orientación Profesional: Nuevos escenarios y perspectivas (pp. 221-241). Madrid: Editorial Biblioteca Nueva.

Bardin, L. (2010). Análise de conteúdo. São Paulo: Editora 70.

Bendassolli, P.F. (2007). Trabalho e identidade em tempos sombrios: Insegurança ontológica na experiência atual com o trabalho. Aparecida: Ideias \& Letras.

Benites, L.C. y Neto, S.S. (2011). Educação física, professores e estudantes: A escolha da docência como profissão e os saberes que lhe são constitutivos. Pensar a Prática, 14(2), 1-11. $<$ https://doi.org/10.5216/rpp.v14i2.12111>

Benites, L.C.; SARTi, F.M. y Neto, S.S. (2015). De mestres de ensino a formadores de campo no estágio supervisionado. Cadernos de Pesquisa, 45(155), 100-117. $<$ http://dx.doi.org/10.1590/198053142928>

CAPES (2010). Ministério da Educação: Plano Nacional de Pós-Graduação - PNPG (2011-2020). Brasilia: CAPES. Recuperado de <http://capes.gov.br/sala-deimprensa? catid $=0 \&$ id $=4439>$.

FigueRA, M.P. (1996). La inserción del universitario en el mercado de trabajo. Barcelona: Ediciones Universidad de Barcelona.

Figuera, M.P.; Buxarrais, M.R.; Llanes, J. y Venceslao, M. (2018). Perfil, motivación y satisfacción académica en los estudiantes de máster: El caso de Ciencias Sociales y Jurídicas. Estudios sobre Educación, 34, 219-237. $<$ https://doi.org/10.15581/004.34.219-237>

HausCHILDT, K. (2018). How are students' study choices related to their social-economic background? Conferencia presentada en el Seminari Internacional de l'Observatori de l'Estudiant «Repensant la formació universitària». Barcelona, 22 de junio de 2018.

Hurtado, S. (2018). From microscope to telescope: Examining students life and university educational practices. Conferencia presentada en el Seminari Internacional de l'Observatori de l'Estudiant «Repensant la formació universitària». Barcelona, 22 de junio de 2018.

Íñguez, T.; Elboj, C. y Valero, D. (2016). La Universidad del Espacio Europeo de Educación Superior ante el abandono de los estudios de grado: Causas y propuestas estratégicas de prevención. Educar, 52(2), 285-313. <https://doi.org/10.5565/rev/educar.674>

KunZer, A.Z. (2005). Políticas de inclusão pelo trabalho, cultura e etnia. En UNISINOS. Anais do II Colóquio Internacional da Cátedra UNESCO-UNISINOS (pp. 1-15). São Leopoldo: UNISINOS. 
Luna, I.N.; Santos, M.M. y Bardagi, M.P. (2014). O desafio da orientação profissional com adolescentes no contexto da modernidade líquida. Revista Ciências Humanas, 48(2), 263-281. <https://doi.org/10.5007/2178-4582.2014v48n2p303>

Méndez Rebolledo, T.J. (2018). La producción académica de los egresados del posgrado de instituciones de educación superior en México. Educar, 54(2), 261-282. <https://doi.org/10.5565/rev/educar.897>

Morosini, M.C. (2009). A pós-graduação no Brasil: formação e desafios. RAES: Revista Argentina de Educación Superior, 1(1), 125-152.

Pochmann, M. (2006). Educação e rendimento do trabalho. Revista Fórum, 35(1), 35-42.

Rodríguez-Moreno, M.L. (coord.); Álvarez, G.M.; Figuera Gazo, M.P. y RodríGUEZ-Espinar, S. (2008). De los estudios universitarios al mundo del trabajo: La construcción del proyecto profesional. Barcelona: Universitat de Barcelona.

Schultz, T. (1963). The economic value of education. Nueva York: Columbia University Press.

Silva, T.C. y Bardagi, M.P. (2015). O aluno de pós-graduação stricto sensu no Brasil: Revisão da literatura dos últimos 20 anos. Revista Brasileira de Pós-Graduação, 12(29), 683-714. <http://dx.doi.org/10.21713/2358-2332.2015.v12.853>

Valério, C. y Souza, S. (2012). Atratividade da carreira docente no curso de pedagogia: Dilemas, escolhas e inserção profissional. Comunicación presentada en el XXIV Congresso de Iniciação Científica da UNESP. São Paulo, 18-19 de septiembre de 2012.

Velloso, A. (2014). A pós-graduação no Brasil: Legados e desafios. Almanaque Multidisciplinar de Pesquisa, 1(1), 4-15. 